\title{
Nurse-Led Behavioral Management of Diabetes and Hypertension in Community Practices: A Randomized Trial
}

\author{
David Edelman, $M D^{1,2}$, Rowena J. Dolor, $M D^{1,3}$, Cynthia J. Coffman, $P h D^{2,4}$, \\ Katherine C. Pereira, PhD ${ }^{5}$, Bradi B. Granger, PhD ${ }^{6}$, Jennifer H. Lindquist, MStat ${ }^{2}$, Alice M. Neary, RN ${ }^{7}$, \\ Amy J. Harris, $B S^{2}$, and Hayden B. Bosworth, $P h D^{1,2}$
}

\begin{abstract}
'Division of General Internal Medicine, Department of Medicine, Duke University Medical Center, Durham, NC, USA; ${ }^{2}$ Center for Health Services Research in Primary Care, Durham VA Medical Center, Durham, NC, USA; ' ${ }^{3}$ Division of General Internal Medicine, Department of Medicine, Vanderbilt Medical School, Nashville, TN, USA; ${ }^{4}$ Department of Biostatistics and Bioinformatics, Duke University Medical Center, Durham, NC, USA; ${ }^{5}$ School of Nursing, Duke University Medical Center, Durham, NC, USA; ${ }^{6}$ Duke Translational Nursing Institute, Duke University Medical Center, Durham, NC, USA.
\end{abstract}

BACKGROUND: Several trials have demonstrated the efficacy of nurse telephone case management for diabetes (DM) and hypertension (HTN) in academic or vertically integrated systems. Little is known about the real-world potency of these interventions.

OBJECTIVE: To assess the effectiveness of nurse behavioral management of DM and HTN in community practices among patients with both diseases.

DESIGN: The study was designed as a patient-level randomized controlled trial.

PARTICIPANTS: Participants included adult patients with both type $2 \mathrm{DM}$ and HTN who were receiving care at one of nine community fee-for-service practices. Subjects were required to have inadequately controlled DM (hemoglobin Alc [Alc] $\geq 7.5 \%$ ) but could have well-controlled HTN.

INTERVENTIONS: All patients received a call from a nurse experienced in DM and HTN management once every two months over a period of two years, for a total of 12 calls. Intervention patients received tailored DM- and HTN- focused behavioral content; control patients received non-tailored, non-interactive information regarding health issues unrelated to DM and HTN (e.g., skin cancer prevention).

MAIN OUTCOMES AND MEASURES: Systolic blood pressure (SBP) and Alc were co-primary outcomes, measured at 6,12 , and 24 months; 24 months was the primary time point.

RESULTS: Three hundred seventy-seven subjects were enrolled; 193 were randomized to intervention, 184 to control. Subjects were $55 \%$ female and $50 \%$ white; the mean baseline Alc was $9.1 \%(\mathrm{SD}=1 \%)$ and mean SBP was $142 \mathrm{mmHg}(\mathrm{SD}=20)$. Eighty-two percent of scheduled interviews were conducted; $69 \%$ of intervention patients and $70 \%$ of control patients reached the 24-month time point. Expressing model estimated differences as (intervention - control), at 24 months, intervention patients had similar Alc [diff=0.1 \%, $95 \%$ CI $(-0.3,0.5), p=0.51]$ and SBP [diff $=-0.9 \mathrm{mmH}$ g, 95\% CI $(-5.4,3.5), p=0.68$ ] values compared to control patients. Likewise, DBP

Registration: ClinicalTrials.gov NCTO0978835

Received May 19, 2014

Revised November 18, 2014

Accepted December 15, 2014

Published online January 8, 2015 (diff=0.4 mmHg, $p=0.76)$, weight (diff=0.3 kg, $p=0.80)$, and physical activity levels (diff=153 MET-min/week, $p=0.41$ ) were similar between control and intervention patients. Results were also similar at the 6- and 12month time points.

CONCLUSIONS: In nine community fee-for-service practices, telephonic nurse case management did not lead to improvement in Alc or SBP. Gains seen in telephonic behavioral self-management interventions in optimal settings may not translate to the wider range of primary care settings.

KEY WORDS: Diabetes; Hypertension; Implementation.

J Gen Intern Med 30(5):626-33

DOI: $10.1007 / \mathrm{s} 11606-014-3154-9$

(C) Society of General Internal Medicine 2015

\section{INTRODUCTION}

Approximately $75 \%$ of all patients with diabetes have concurrent hypertension (HTN). ${ }^{1}$ While blood sugar control is the focal point of diabetes management for most patients, data suggest that there may be more benefit in blood pressure (BP) control than reduction of blood sugar in patients with diabetes. ${ }^{2}$ Evidence from several randomized controlled trials $(\mathrm{RCTs})^{3-5}$ has demonstrated reduced microvascular and cardiovascular complications when BP is tightly controlled. It has been estimated that rigorous control of BP is substantially more cost-effective than similarly tight blood sugar control in patients with diabetes, and is potentially cost-saving. ${ }^{2}$

Nurse management programs for diabetes have been formally tested in randomized controlled trials in a handful of academic settings, and have been efficacious in controlling glycemia. ${ }^{6-9}$ In these studies, changes in hemoglobin A1c (A1c) levels have reached as high as 1.1 percentage points. ${ }^{6-10}$ These studies have been conducted in settings with strong academic affiliations, where intervention delivery can be tightly controlled; the potency of interventions under these ideal settings is generally referred to as "efficacy." In contrast, "effectiveness" refers to the potency of interventions under circumstances close to those that would be experienced in medical practice. It is generally assumed that some potency will be lost in assessing the effectiveness of efficacious 
interventions, but there are few trials that have measured this decrement directly, and none in diabetes nurse management. Additionally, trials in nurse management of HTN were conducted in similar settings. ${ }^{10,11}$ There are no randomized trial data on the effectiveness of behavioral co-management of diabetes and HTN.

Our objective in this randomized controlled trial was to assess the effectiveness of nurse-assisted behavioral comanagement of blood sugar and BP among patients with diabetes and HTN in a practice-based research network of community primary care practices.

\section{METHODS}

Study Design. The study was a single-blinded randomized controlled trial (RCT) of a tailored telephone-delivered behavioral nurse intervention. A detailed description of the methods utilized in the Tailored Case Management for Diabetes and Hypertension (TEACH-DM) study were published previously. ${ }^{12}$

Setting. We performed the study in nine primary care practices in the Duke Clinical Research Institute Primary Care Research Consortium (PCRC). Practices in this established primary care-based research network are only very loosely academically affiliated; only two of the 71 providers across our nine clinics have any teaching responsibilities with Duke (limited to one half-day in an outpatient setting). Practices comprised both physician and mid-level primary care providers, trained in either general internal medicine or family medicine, and located in either urban or rural settings.

Patients. Patients had been enrolled in primary care at one of the nine clinics for at least a year at the time of enrollment. Eligible patients were identified through administrative data, and a list of eligible patients was generated for each PCP. The lists were vetted by each PCP for participation of individual patients. Patients were required to have both diabetes and HTN, and had to be taking medication for both, as confirmed by administrative data as well as patient selfreporting. Blood sugar had to be inadequately controlled, as determined by most recent $\mathrm{A} 1 \mathrm{c} \geq 7.5 \%$, but subjects were not required to have poorly controlled BP. Patients were broadly excluded for any of a range of comorbid serious illnesses ${ }^{12}$, type I diabetes, the inability to receive a telephone intervention in English, participation in another diabetes or HTN study, or living in an assisted living facility.

Trial Protocol. After patients had provided written informed consent, we obtained baseline measurements including height, weight, A1c, BP, and demographics from all patients. Patients remaining eligible (i.e., with $\mathrm{A} 1 \mathrm{c} \geq 7.5 \%$ ) were randomized to intervention or control groups at a 1:1 ratio, stratified by clinic and baseline BP control (systolic BP $[\mathrm{SBP}] \geq 130$ or diastolic
$\mathrm{BP}[\mathrm{DBP}] \geq 80$ vs. SBP $<130$ and $\mathrm{DBP}<80$ ). Regardless of arm assignment, all subjects had follow-up ascertainment of all outcomes at 6,12 , and 24 months after enrollment by a research assistant blinded to arm assignment.

Outcome Measurement. A1c and BP were measured using standard methods. A1c was measured by fingerstick on a wellvalidated point-of-care A1c machine (Bayer DCA 2000, ${ }^{13}$ ), calibrated to the national standard (mean $=5.0 \%$, top of normal range $=6.0 \%$ ). For BP, at each visit, two measures at 5-min intervals were obtained and were then averaged for the outcome. ${ }^{14} \mathrm{SBP}$ and A1c were co-primary outcomes; DBP and $\mathrm{BP}$ control (SBP $<130$ and $\mathrm{DBP}<80$, as per guidelines at the start of the study) were secondary outcomes.

Other key secondary outcomes included weight and physical activity. Weight was measured using the same scale for each patient at all visits. Physical activity was measured by means of the short-form International Physical Activity Questionnaire (IPAQ), a validated measure of leisure-time physical activity, the outcome of which is expressed in metabolic equivalents. ${ }^{15}$ Covariates included age, race, ethnicity, gender, education, financial security, self-efficacy, health literacy, and medication adherence. Self-efficacy was measured with the Perceived Competence Scale, ${ }^{16}$ health literacy was measured using the Rapid Estimate of Adult Literacy in Medicine (REALM) tool, ${ }^{17}$ and medication adherence was obtained from self-reports and dichotomized into perfect vs. imperfect. ${ }^{18}$

Intervention. A single nurse with extensive experience in case management delivered both the tailored behavioral intervention and the attention control. For both arms, calls were delivered every two months, for a total of 12 calls.

For the intervention arm, the nurse's behavior-modifying content was tailored to each patient's individual barriers to controlling blood sugar or BP. This content was divided into a series of topical modules addressing one or more behaviors appropriate for improving control of BP or blood sugar, and included physical activity, weight reduction, low salt intake, smoking cessation, medication adherence, management of hypoglycemia, and blood glucose monitoring. The full list of modules was published previously. ${ }^{12}$ These modules were activated only if a patient required them (e.g., nonsmokers did not receive smoking cessation advice). The modules assessed barriers to specific behaviors, and the nurse then tried to engage the patient in problem-solving in order to determine actions for overcoming these barriers. In addition, barriers that might generalize to a number of problems - specifically, low levels of disease knowledge, poor memory, poor social support, and concern about the quality of physician-patient decisionmaking - were addressed on their own. Fidelity was assessed by two nurse-investigators (KP, BG), who listened to a sample of $5 \%$ of total calls for delivery of intended content.

The control patients received calls that were not tailored; these calls provided traditional didactic information on a range 
of topics that had no relationship to HTN, DM, or any of the behaviors we were trying to improve (e.g., flu shots, skin cancer prevention). Content was tightly scripted, designed to limit the potential for productive interaction between nurse and patient, and was informed by standard guidelines as stated on government websites.

Analysis. Sample Size and Power Considerations. The original proposed primary outcomes (BP control and glycemic control) were longitudinal and binary. The sample size estimate was based on the second primary hypothesis that patients in the intervention group would achieve a higher rate of adequate BP control compared to the attention control group over a two-year follow-up period. The BP hypothesis was chosen because it required a larger sample than the glycemic control hypothesis (because not all patients had poorly controlled BP, while all had poorly controlled A1c). Assuming a $40 \%$ baseline rate of control, a type I error rate of $5 \%$, a dropout rate of $20 \%$, and an $\operatorname{AR}\left({ }^{1}\right)$ correlation structure, we estimated that 200 subjects in each arm had $80 \%$ power to detect a $20 \%$ change in proportion of BP control over 24 months, for a total of 400 subjects. Before enrollment began, however, a published study suggested that the HbAlc level was best analyzed as a continuous variable, ${ }^{19}$ and so we decided at that time to analyze our primary outcomes as continuous A1c and SBP. The sample size of 200 patients per arm was projected to yield greater than $80 \%$ power to detect clinically meaningful differences in these outcomes.

Participants were analyzed in the group to which they were assigned regardless of whether they participated in any intervention calls. ${ }^{20}$ Statistical analyses were performed using SAS for Windows (version 9.2; SAS Institute, Cary, NC, USA). For primary analysis, we fit linear mixed models (LMM) with a random effect for clinic and an unstructured covariance model for the repeated measures. ${ }^{21}$ Our primary predictors included indicator variables for the 6-, 12-, and 24-month follow-up periods and interaction variables for the treatment group by follow-up time; primary inference was on treatment by 24 month follow-up interaction. All available patient data were used; no observations were deleted because of missing followup data. LMM via maximum likelihood estimation implicitly accommodates missingness when missingness is due to treatment, prior outcome, or other baseline covariates included in the model, defined as missing at random. ${ }^{22}$ The final models included a stratification variable for baseline BP control (SBP $\geq 130$ or $\mathrm{DBP} \geq 80$ vs. $\mathrm{SBP}<130$ and $\mathrm{DBP}<80$ ). For sensitivity or per-protocol analyses, we fit the same LMM as described above to the subgroup of subjects that completed at least 10 intervention or attention control calls. The secondary continuous outcomes of DBP, weight, and metabolic equivalent of task (MET) minutes per week were analyzed using LMM as described above for the primary outcomes. For sensitivity analysis, we transformed MET minutes per week using a square root transformation, and we also compared the change in MET minutes from baseline to 24 months between arms, using a Wilcoxon rank-sum test. For the secondary binary outcome, BP control, we used a generalized estimating equation (GEE) model, ${ }^{23}$ which accounts for clustering of patients within clinics and repeated measures within patients. We used a logit link and unstructured covariance.

\section{RESULTS}

Patients. We randomly assigned 377 of the 2,601 patients assessed for eligibility (Fig. 1). Most of the ineligible patients were excluded because of improved HbAlc control; serious comorbid illness also accounted for some exclusions. We enrolled patients between June 2009 and July 2011. Seventy-eight percent of patients completed the 12-month follow-up, and 263 patients (70\%) reached the primary 24month endpoint. Overall, we completed $82 \%$ of interviews. These 377 patients were enrolled from nine practices, with as many as 104 and as few as 16 patients from a single practice. Patient characteristics in the intervention and control arms were similar at baseline (Table 1).

Attendance. Table 2 shows the distribution of completed encounters by randomization arm. Patients in the intervention arm completed $78 \%$ of scheduled calls; control patients completed $81 \%$. The mean (SD) number of completed calls (of a total of 12 scheduled) was 9.7 (3.5) for control and 9.3 (3.3) for intervention patients. The mean number of completed calls was similar between arms $(p=0.23)$.

Primary Outcomes. After adjustment for stratification variables, the estimated mean A1c and SBP were similar between arms at the primary endpoint of 24 months (intervention $0.1 \%$ higher than control, $95 \%$ CI $[-0.3 \%, 0.5 \%], p=0.50$ for A1c; intervention $0.9 \mathrm{mmHg}$ lower than control, $95 \% \mathrm{CI}[-5.4$, 3.5], $p=0.69$ for SBP; see Figs. $2 \mathrm{a}$ and b). Similarly, we found no difference between arms in mean A1c at $6(p=0.65)$ or 12 months $(p=0.23)$, or in mean SBP at $6(p=0.10)$ or 12 months $(p=0.54)$ (Fig. 2a). The largest estimated difference in SBP between arms was at 6 months (intervention $3.3 \mathrm{mmHg}$ lower than control). The estimated intraclass correlation coefficients (ICC) were 0.008 for A1C and 0.002 for SBP. In sensitivity analyses of patients who completed at least 10 intervention or attention control calls, similar results were found for both HbAlc and SBP (not shown).

Secondary Outcomes. We analyzed several secondary outcomes. Briefly, there were no significant differences between arms in DBP, weight, or physical activity throughout the two-year study period (see Table 3). At 24 months, the 


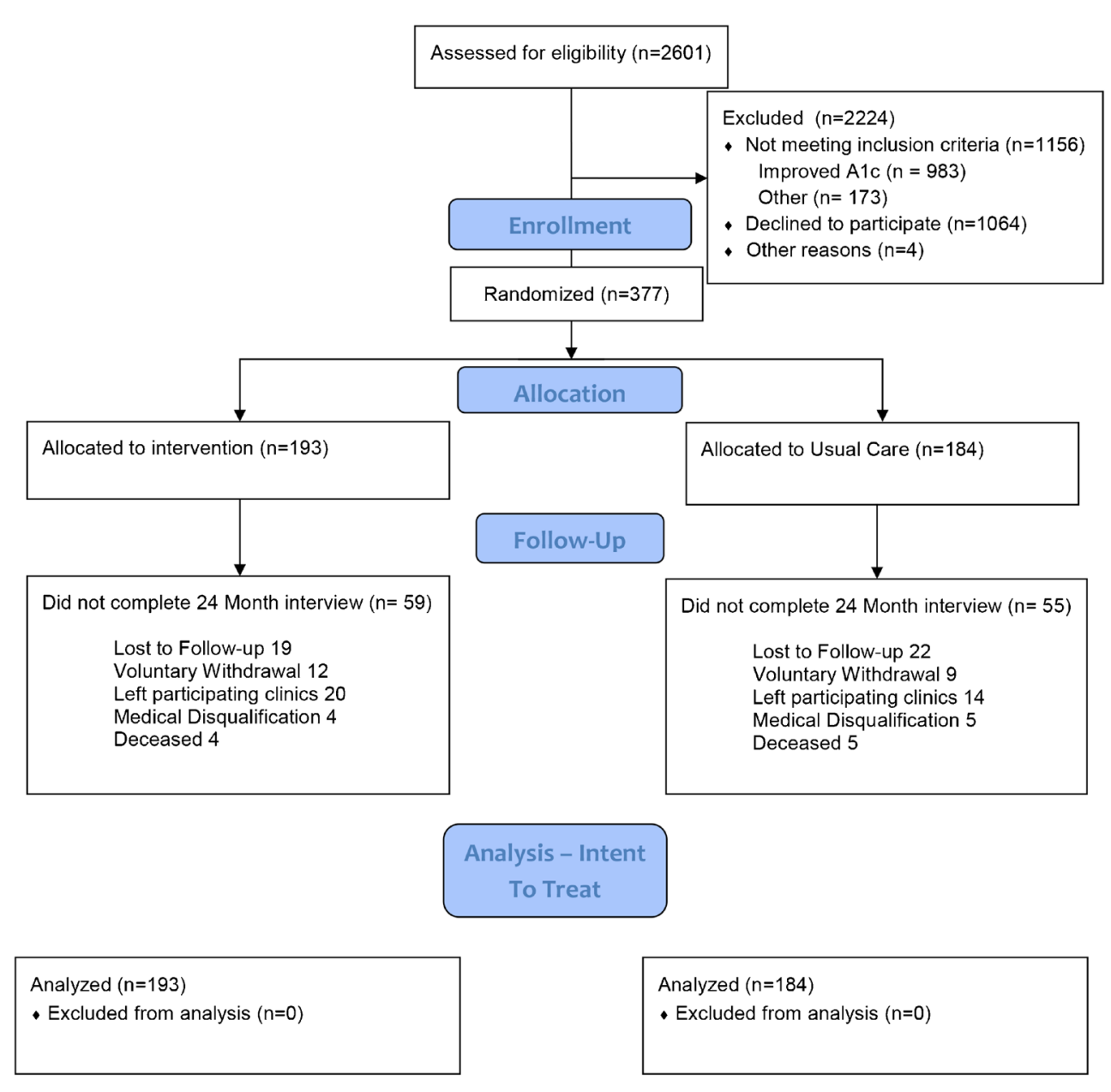

Fig. 1 CONSORT diagram for the trial

difference in DBP between arms was $0.4 \mathrm{mmHg}$ (lower in the control arm), the mean weight was $0.3 \mathrm{~kg}$ higher among intervention group patients, and mean leisure time physical activity was $150 \mathrm{MET}$-min/week higher for intervention versus control patients. Similar results were found with a square root transformation applied to the leisure time activity outcome, and we found no difference between arms in change in leisure time physical activity at 24 months using a Wilcoxon sum test $(p=0.14)$. Finally, we found no difference between arms in BP control rates (OR 1.1, $95 \% \mathrm{CI}[0.6,2.0] ; p=0.80$ ), with estimated BP control rates of $18.3 \%$ for the intervention arm and $17.2 \%$ for the control arm at 24 months.

\section{DISCUSSION}

A tailored case management intervention for diabetes and hypertension in a community practice setting failed to yield improvements in SBP or A1c among patients. This null finding was as a result of an improvement in A1c levels for both the control and intervention arms, and a lack of improvement in SBP values for either arm, and occurred despite our intervention having previously shown efficacy for BP in an academic setting ${ }^{10,24}$ and similar interventions having shown efficacy for A1c in other academic settings. This result leads to the question of whether the intervention failed to translate to the community setting because of differences inherent in the complexities of translating interventions to community settings in general or our practices in particular, or because of some other concern.

It is important to consider the possibility that our intervention in particular, and nurse case management in general, does not work very well in community settings. Previous trials have been conducted in VA facilities, resident teaching clinics, and other highly controlled settings, with teaching physicians and less pressure to generate revenue. The practices we used had very few $(2 / 71)$ teaching primary care providers, and they were fee-for-service providers whose primary purpose beyond excellent care is to generate revenue. Moreover, we are not alone in experiencing this difficulty in community practices; since the end of our trial, three trials have been published of similar interventions in community practices in three countries in Europe, all of which showed no impact on clinical outcomes. ${ }^{25-27}$

The purpose of our trial was to rigorously evaluate an intervention similar to nurse behavioral management interventions that have almost certainly been implemented in a number 
Table 1 Subject Characteristics, Overall and by Study Arm

\begin{tabular}{|c|c|c|c|}
\hline Demographic $^{a}$ & Total $(N=377)$ & Intervention $(N=193)$ & Control $(N=184)$ \\
\hline Age, mean (SD) & $58.7(10.9)$ & $57.8(10.9)$ & $59.6(10.7)$ \\
\hline Male gender & $45.4 \%$ & $45.6 \%$ & $45.1 \%$ \\
\hline Non-white race & $50.1 \%$ & $50.8 \%$ & $49.5 \%$ \\
\hline \multicolumn{4}{|l|}{ Marital status } \\
\hline Married & $60.2 \%$ & $58.5 \%$ & $62.0 \%$ \\
\hline \multicolumn{4}{|l|}{ Education } \\
\hline High school or less & $49.1 \%$ & $47.2 \%$ & $51.1 \%$ \\
\hline \multicolumn{4}{|l|}{ Employment status } \\
\hline Full-time & $38.2 \%$ & $39.9 \%$ & $36.4 \%$ \\
\hline Part-time & $5.8 \%$ & $2.6 \%$ & $9.2 \%$ \\
\hline Not employed/retired & $54.9 \%$ & $56.5 \%$ & $53.3 \%$ \\
\hline \multicolumn{4}{|l|}{ Financial situation } \\
\hline Can pay bills only by cutting spending, & $41.7 \%$ & $38.3 \%$ & $45.1 \%$ \\
\hline $\begin{array}{l}\text { or cannot always pay bills } \\
\text { Low health literacy }(<60 \text { on REALM })\end{array}$ & $316 \%$ & $316 \%$ & \\
\hline \multicolumn{3}{|l|}{ Clinical data } & $31.5 \%$ \\
\hline Alc $(\%)$, mean $(\mathrm{SD})$ & $9.1(1.5)$ & $9.2(1.5)$ & $9.0(1.4)$ \\
\hline $\mathrm{SBP}(\mathrm{mmHg})$, mean (SD) & $142.2(20.3)$ & $142.0(20.7)$ & $142.5(20.0)$ \\
\hline DBP (mmHg), mean (SD) & $80.7(11.4)$ & $81.5(11.6)$ & $79.8(11.0)$ \\
\hline BP control (SBP <130 and DBP <80) & $19.6 \%$ & $19.2 \%$ & $20.1 \%$ \\
\hline Weight $(\mathrm{kg})$, mean (SD) & $105.3(24.2)$ & $104.5(23.7)$ & $106.1(24.6)$ \\
\hline BMI (kg/m²), mean (SD) & $36.3(7.7)$ & $36.2(7.9)$ & $36.4(7.5)$ \\
\hline Total MET min per week, median (IQR) & $198.0(0.706)$ & $150.8(0.503)$ & $247.5(0.836)$ \\
\hline Perceived Competence Scale, mean (SD) & $2.5(0.8)$ & $2.5(0.8)$ & $2.4(0.8)$ \\
\hline Non-med-adherent (med-taking scale) BP & $29.2 \%$ & $26.9 \%$ & $31.5 \%$ \\
\hline Non-med-adherent (med-taking scale) DM & $43.0 \%$ & $43.0 \%$ & $42.9 \%$ \\
\hline
\end{tabular}

${ }^{a}$ REALM is missing for five patients, and one patient is missing for each of the Perceived Competence Scale and the total MET-min per week

of community practices. These interventions likely show apparent efficacy in uncontrolled settings, thus encouraging practices to continue to devote resources to nurse management. However, the improvement in Alc seen in the control arm of our study underscores the fallacy in assuming that it was the case management that led to the improvement in these cases. Patients who are eligible to start a behavioral intervention at the nadir of their disease control are likely to improve over time, with or without the program, and for that reason, controlled trials are better positioned to measure the value of such programs. ${ }^{28}$ The null result of this trial, therefore, supports the possibility that the apparent gains of nurse management programs are due to this regression to the mean, and that these interventions may be ineffective. However, there are a few categories of other possible reasons for our null result.

There are at least three important design elements of this trial that may have portended the negative result. First, we used an attention control; most previous studies of disease management for DM and/or HTN have used usual care controls (including our previous studies in HTN). The reason for attention controls is precisely the concern that contact time with the patient, independent of content of intervention, may be a potent intervention, and theoretically this could explain the Alc result. However, many studies have shown that didactic lectures on diabetes-related topics are a weak interventional approach (reviewed $\mathrm{in}^{29}$ ), much less lectures on unrelated information. Also, a potent attention control does not explain the lack of improvement in BP in either arm. Second, co-intervention is always a concern in effectiveness trials of quality improvement (QI) type interventions, and a cointervention in the form of QI initiatives in the practice network theoretically could have caused the null result. Again, this is not a likely explanation for the BP results, where there was no improvement at all. Additionally, the mean A1c for patients in Duke Primary Care was unchanged at $7.3 \%$ over the course of the study, suggesting that system-wide co-intervention was not an important factor for improvement in A1c among the control group. Finally, our choice to allow patients to enter the study despite having adequate BP control may have contributed to the null $\mathrm{BP}$ result. We made this decision based on the fact that $40 \%$ of patients who are in control at a given moment fall out of control within a year, ${ }^{30}$ and the mean baseline SBP was well above the treatment goal of $130 \mathrm{mmHg}$ for patients with diabetes in essentially all guidelines used for the duration of the study; $72 \%$ of patients had baseline SBP > $130 \mathrm{mmHg}$. Furthermore, this is not an explanation for the A1c results.

Table 2 Number of Completed Intervention Calls by Arm

\begin{tabular}{|c|c|c|c|c|c|c|c|c|c|c|c|c|c|c|}
\hline & \multicolumn{14}{|c|}{ Number of successful encounters } \\
\hline & $\mathbf{0}$ & 1 & 2 & 3 & 4 & 5 & 6 & 7 & 8 & 9 & 10 & 11 & 12 & Total \\
\hline Control & 2 & 5 & 9 & 4 & 7 & 2 & 6 & 5 & 6 & 5 & 12 & 17 & 104 & 184 \\
\hline Intervention & 0 & 1 & 5 & 14 & 10 & 7 & 8 & 10 & 6 & 8 & 17 & 23 & 84 & 193 \\
\hline
\end{tabular}



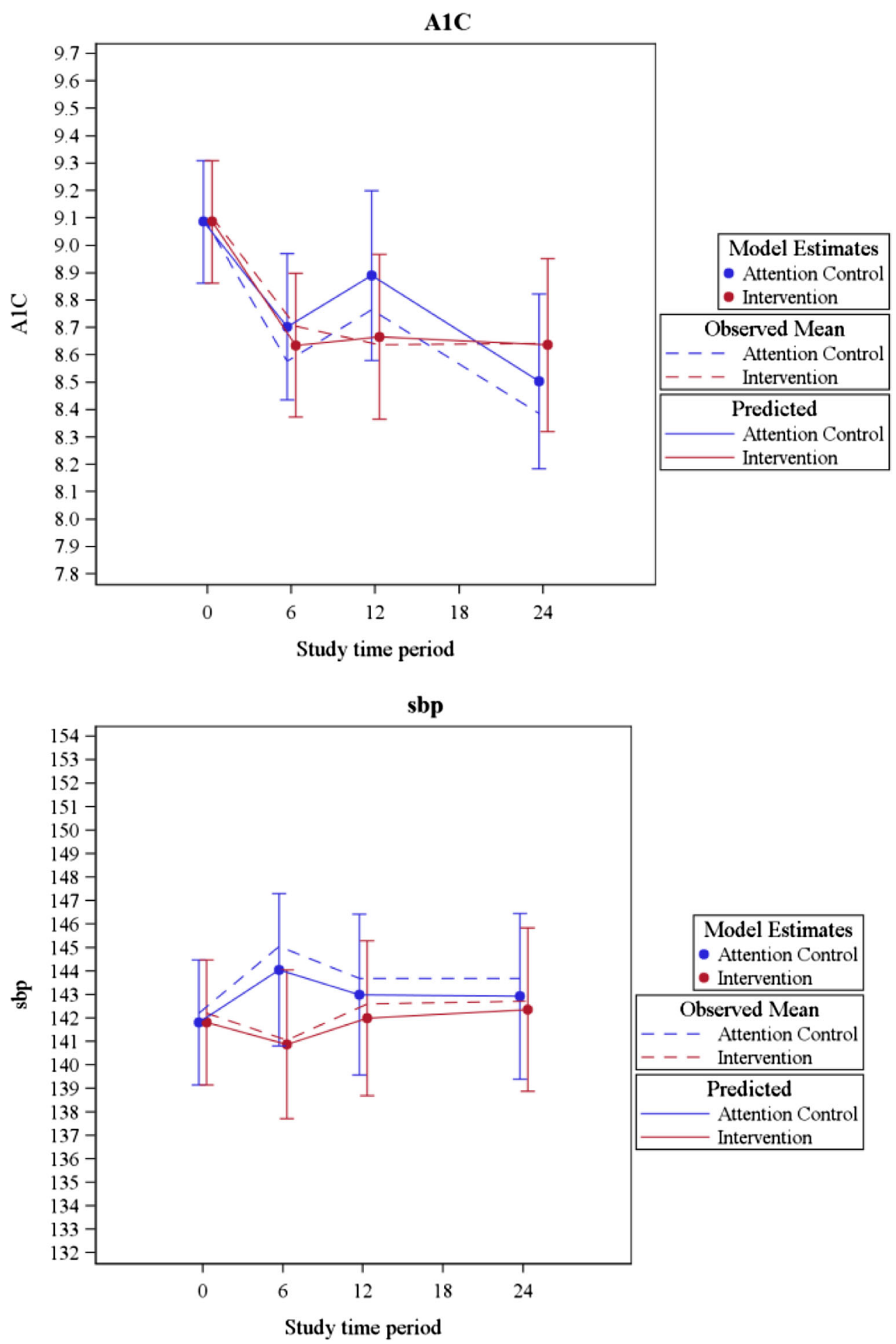

Fig. 2 Hemoglobin A1c (A1c, a) and systolic blood pressure (SBP, b) by study arm over time

It is also possible that our intervention was not just ineffective, but non-efficacious, despite the prior literature suggesting efficacy. Part of the challenge of working in community practices is that it is very hard to closely link an intervention or interventionist with any of the several practices within a study, and any such linkage was frankly considered undesirable by many of the practices. This lack of integration with primary care may have weakened the intervention; this has been a concern for other telephone management interventions as well. ${ }^{31}$ It is also possible that, while the telephone was an important modality for intervention delivery at the time that prior studies were conducted, patients now prefer web- or text- 
Table 3 Estimated Mixed Model-Derived Difference in Secondary Outcomes Between Intervention and Control Arms

\begin{tabular}{llc}
\hline \hline Outcome & $\begin{array}{l}\text { Time of } \\
\text { measurement }\end{array}$ & $\begin{array}{l}\text { Intervention vs. control } \\
(\mathbf{9 5} \% \mathbf{C I}), \boldsymbol{p} \text { value }\end{array}$ \\
\hline DBP (mmHg) & 6 months & $1.3(-1.0,3.5), 0.27$ \\
& 12 months & $1.0(-1.1,3.1), 0.36$ \\
& 24 months & $0.4(-2.1,2.9), 0.76$ \\
Weight (kg) & 6 months & $0.1(-1.5,1.8), 0.87$ \\
& 12 months & $1.1(-0.6,2.7), 0.21$ \\
Activity (MET-min/ & 24 months & $0.3(-1.9,2.4), 0.80$ \\
week) & 6 months & $76(-219,370), 0.61$ \\
& 12 months & $133(8990,456), 0.42$ \\
& 24 months & $153(-212,519), 0.41$ \\
\hline
\end{tabular}

based interventions, and the telephone is no longer a potent means of intervention delivery.

Regarding the BP outcome, we have had extensive experience with this part of the intervention, and we do not think that our content was inadequate to effect BP lowering. In contrast, we had not previously developed a DM intervention. And while the DM intervention was built around the same theoretical principles as the HTN intervention, and contained the same domains of material as were used in other similar interventions in the literature, it is possible that the DM-focused content of the intervention (e.g., self-monitoring, insulin management) was not as potent as in other studies. It is also possible that the two-month frequency of contact that had worked for us in HTN was not frequent enough to produce a change in $\mathrm{Alc}$; prior diabetes interventions have used monthly calls. ${ }^{9}$

There are two analytic issues that must also be considered as possible contributing factors. First, the study may have been underpowered for the BP outcome objective. Sample standard deviations (SDs) are unpredictable. Our previous work had shown SD ranging from 14 to $17 \mathrm{mmHg},{ }^{10,32}$ but our SD in this study was almost $21 \mathrm{mmHg}$. With this unexpectedly large $\mathrm{SD}$, a relatively small absolute effect size of $<0.25 \mathrm{SD}$ becomes clinically important, and our $95 \%$ CIs around the difference in SBP between arms fail at all time points to exclude clinically important effects favoring intervention $(\sim 5 \mathrm{mmHg})$. This is a lesser consideration for A1c, where at two of three time points, $95 \%$ CIs exclude clinically relatively small differences of $0.35 \%$ favoring intervention. Unexpectedly high rates of dropout (30\% at 24 months, compared to $20 \%$ expected) also contributed to the limited power. Second, the results of this study may simply reflect a spurious type II error. Spurious error, by convention, is better tolerated for null results than for positive findings (i.e., $\beta$ is set greater than $\alpha$ in power calculations), and it is certainly possible that we randomly enrolled a sample of patients less responsive to disease management than the population as a whole.

Our study illustrates the intertwined challenges associated with both the translation of chronic illness interventions to community practices as well as the scientific study of that translation. While small gains in clinical outcomes may add up to an important public health impact over a large population, the study of a modest intervention by traditional trial methods may not be feasible. Future studies will want to start with more potent interventions, perhaps with more frequent contact, so that their effects can be measured even after potency is lost in translation. Alternatively, they will want to have a greatly increased sample size such that smaller effects among more statistically diverse samples can be detected.

Acknowledgments: The authors wish to thank the Duke Primary Care Research Consortium TEACH-DM clinics (Duke Primary Care [DPC] Butner-Creedmoor, DPC Harps Mill, DPC Henderson, DPC Hillsborough, DPC Wake Forest, Durham Medical Center, Oxford Family Physicians, Sutton Station Internal Medicine, and Triangle Family Practice), as well as Muriel Dorvilier, Heather Webb, and Amy Edens. This research was supported by grant number R01DK074672 from the National Institute of Diabetes and Digestive and Kidney Diseases (NIDDK). Data from this presentation were presented at the Society for General Internal Medicine in April 2014, and at AcademyHealth in June 2014.

Conflict of Interest: The authors each declare that they have no conflict of interest.

Corresponding Author: David Edelman, MD; Center for Health Services Research in Primary CareDurham VA Medical Center, 508 Fulton St (152), Durham, NC 27705, USA (e-mail: dedelman@duke.edu).

\section{REFERENCES}

1. McFarlane SI, Jacober SJ, Winer N, Kaur J, Castro JP, Wui MA, Gliwa A, Von Gizycki H, Sowers JR. Control of cardiovascular risk factors in patients with diabetes and hypertension at urban academic medical centers. Diabetes Care. 2002;25(4):718-23.

2. The CDC Diabetes Cost Effectiveness group. Cost-effectiveness of intensive glycemic control, intensified hypertension control, and serum cholesterol level reduction for type 2 diabetes. JAMA. 2002;287:2542-51.

3. Hansson L, Zanchetti A, Carruthers SG, Dahlof B, Elmfeldt D, Julius S, Menard J, Rahn KH, Wedel $\mathbf{H}$, Westerling $\mathbf{S}$. Effects of intensive bloodpressure lowering and low-dose aspirin in patients with hypertension: principal results of the Hypertension Optimal Treatment (HOT) randomised trial. Lancet. 1998;351(9118):1755-62.

4. Estacio RO, Jeffers BW, Gifford N, Schrier RW. Effect of blood pressure control on diabetic microvascular complications in patients with hypertension and type 2 diabetes. Diabetes Care. 2000;23(S2):B54-64.

5. Adler AI, Stratton IM, Neil HA, Yudkin JS, Matthews DR, Cull CA, Wright AD, Turner RC, Holman RR. Association of systolic blood pressure with macrovascular and microvascular complications of type 2 diabetes (UKPDS 36): prospective observational study. BMJ. 2000;321(7258):412-9.

6. Aubert RE, Herman WH, Waters J, Moore W, Sutton D, Peterson BL, Bailey CM, Koplan JP. Nurse case management to improve glycemic control in diabetic patients in a health maintenance organization. A randomized, controlled trial. Ann Intern Med. 1998;129(8):605-12.

7. Sadur CN, Moline N, Costa M, et al. Diabetes management in a health maintenance organization. Diabetes Care. 1999;22(12):2011-7.

8. Thompson DM, Kozak SE, Sheps S. Insulin adjustment by a diabetes nurse educator improves glucose control in insulin-requiring diabetic patients: a randomized trial. CMAJ. 1999;161(8):959-62.

9. Weinberger M, Kirkman MS, Samsa GP, Shortliffe EA, Landsman PB, Cowper PA, Simel DL, Feussner JR. A nurse-coordinated intervention for primary care patients with non-insulin-dependent diabetes mellitus: impact on: glycemic control and health-related quality of life. J Gen Intern Med. 1995; 10(2):59-66.

10. Bosworth HB, Powers BJ, Olsen MK, et al. Home blood pressure management and improved blood pressure control: results from a randomized controlled trial. Arch Intern Med. 2011;171(13):1173-80.

11. Bosworth HB, Olsen MK, Grubber JM, et al. Two self-management interventions to improve hypertension control: a randomized trial. Ann Intern Med. 2009;151(10):687-95.

12. Crowley MJ, Bosworth HB, Coffman CJ, et al. Tailored case management for diabetes and hypertension (TEACH-DM) in a community population: study design and baseline sample characteristics. Contemp Clin Trials. 2013;36(1):298-306 
13. Arsie MP, Marchioro L, Lapolla A, et al. Evaluation of diagnostic reliability of DCA 2000 for rapid and simple monitoring of HbAlc. Acta Diabetol. 2000;37(1):1-7.

14. Kim J, Bosworth HB, Voils CI, Olsen MK, Dudley T, Gribbin M, Oddone EZ. How well do clinic-based blood pressure measurements agree with rigorous standards? J Gen Intern Med. 2005;20(7):647-9.

15. Heisler M, Bouknight RR, Hayward RA, Smith DM, Kerr EA. The relative importance of physician communication, participatory decision making, and patient understanding in diabetes self-management. J Gen Intern Med. 2002;17(4):243-52.

16. Davis TC, Long SW, Jackson RH, Mayeaux EJ, George RB, Murphy PW, Crouch MA. Rapid estimate of adult literacy in medicine: a shortened screening instrument. Fam Med. 1993;25(6):391-5.

17. Morisky DE, Green LW, Levine DM. Concurrent and predictive validity of a self-reported measure of medication adherence. Med Care. 1986;24:67-74.

18. Pogach LM, Rajan M, Aron DC. Comparison of weighted performance measurement and dichotomous thresholds for glycemic control in the Veterans Health Administration. Diabetes Care. 2006;29(2):241-6.

19. Group IEEW. ICH harmonised tripartite guideline-statistical principles for clinical trials. Stat Med. 1999; 18:1905-42.

20. Verbeke G, Molenbergh G. Linear mixed models for longitudinal data. New York: Springer; 2000.

21. Little RJA, Rubin DB. Statistical analysis with missing data. New York: Wiley; 2002

22. Diggle PJ, Heagerty P, Liang KY, Zeger SL. Analysis of longitudinal data. Oxford: Oxford Univ Press; 2002.

23. Bosworth HB, Olsen MK, Dudley T, Orr M, Goldstein MK, Datta SK, McCant F, Gentry P, Simel DL, Oddone EZ. Patient education and provider decision support to control blood pressure in primary care: a cluster randomized trial. Am Heart J. 2009; 157(3):450-6.
24. Mons U, Raum E, Kramer HU, et al. Effectiveness of a supportive telephone counseling intervention in type 2 diabetes patients: randomized controlled study. PLoS ONE. 8(10):e77954.

25. Jansink R, Braspenning J, Keizer E, Van Der Weijden T, Elwyn G, Grol R. No identifiable $\mathrm{Hb} 1 \mathrm{Ac}$ or lifestyle change after a comprehensive diabetes programme including motivational interviewing: a cluster randomised trial. Scand J Prim Health Care. 2013;31:119-27.

26. Blackberry ID, Furler JS, Best JD, Chondros P, Vale M, Walker C, Dunning T, Segal L, Dunbar J, Audehm R, Liew D, Young D. Effectiveness of general practice based, practice nurse led telephone coaching on glycaemic control of type 2 diabetes: the Patient Engagement And Coaching for Health (PEACH) pragmatic cluster randomized controlled trial. BMJ. 2013;347:f5272.

27. Stephenson J, Imrie $\mathbf{J}$. Why do we need randomised controlled trials to assess behavioural interventions? BMJ. 1998;316(7131):611-3.

28. Norris SL, Lau J, Smith SJ, Schmid CH, Engelgau MM. Selfmanagement education for adults with type 2 diabetes: a meta-analysis of the effect on glycemic control. Diabetes Care. 2002;25(7):1159-71.

29. Bosworth HB, Dudley TK, Orr M, Neary A, Harrelson M, Adams M, Svetkey LP, Dolor RJ, Oddone EZ. The Take Control of Your Blood pressure (TCYB) study: study design and methodology. Contemp Clin Trials. 2007;28(1):33-47.

30. McCall N, Cromwell J. Results of the medicare health support diseasemanagement pilot program. N Engl J Med. 2011;365:1704-12.

31. Edelman D, Fredrickson S, Melnyk SD, et al. Group medical clinics versus usual care for patients with both diabetes and hypertension. Ann Intern Med. 2010;152:689-96.

32. Craig CL, Marshall AL, Sjostrom M, et al. International physical activity questionnaire: 12-country reliability and validity. Med Sci Sports Exerc. 2003;35(8): 1381-95. 